\title{
A left ventricular segmentation based on a parallel watershed transformation towards an accurate heart function evaluation
}

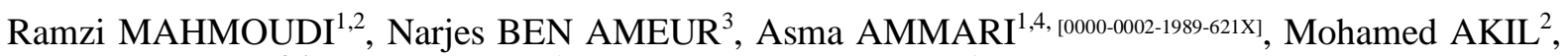 \\ Rachida SAOULI ${ }^{2,4}$, Badii HMIDA ${ }^{5}$, Momahed Hedi BEDOUI ${ }^{1}$ \\ ${ }^{1}$ Faculty of Medicine of Monastir, Medical Imaging Technology Lab - LTIM-LR12ES06, University of Monastir, 5019 \\ Monastir, Tunisia. \\ ${ }^{2}$ Gaspard-Monge computer-science laboratory, Paris-Est University, Mixed Unit CNRS-UMLV-ESIEE UMR8049, BP99, \\ ESIEE Paris Cité Descartes, 93162 Noisy Le Grand, France. \\ ${ }^{3}$ Higher Institute of Medical Technologies of Tunis, Laboratory of Biophysics and Medical Technologies, University of \\ Tunis El Manar, Tunis, Tunisia \\ ${ }^{4}$ Department of computer science, Laboratory of Intelligent Computing (LINFI), Mohamed Khaider University BP 145 RP \\ 07000, Biskra, Algeria \\ ${ }^{5}$ Radiology Service, UR12SP40, CHU Fattouma Bourguiba, 5019 Monastir, Tunisia. \\ *ramzi.mahmoudi@esiee.fr
}

\begin{abstract}
Magnetic Resonance Imaging (MRI) has emerged as the golden reference for cardiac examination. This modality allows the assessment of human cardiovascular morphology, functioning, and perfusion. Although a couple of challenging issues, such as the cardiac MR image's features and the large variability of images among several patients, still influences the cardiac cavities' segmentation and needs to be carried out. In this paper, we have profoundly reviewed and fully compared semi-automated segmentation methods performed on cardiac Cine-MR short-axis images for the evaluation of the left ventricular functions. However, the number of parameters handled by the synthesized works is limited if not null. For the sake of ensuring the highest coverage of the LV parameters computing, we have introduced a parallel watershedbased approach to segment the left ventricular allowing hence the computation of six parameters (End-Diastolic Volume, End-Systolic Volume, Ejection Fraction, Cardiac output, Stroke Volume and Left Ventricular Mass). An algorithm is associated with main considered measurements. The experimental results that were obtained through studying twenty patients' MRI data base, demonstrate the accuracy of our approach for estimating real values of the maximal set of parameters thanks to a faithful segmentation of the myocardium.
\end{abstract}

\section{Introduction}

The heart is considered as one of the most sensitive organs that plays a crucial role in the body [1]. Thus, any cardiacrelated dysfunction might have heavy consequences on the human body such as dyspnea, persistent coughing, build-up of excessive fluid in the body tissues and tiredness [2][3]. Therefore, the diagnosis of cardiovascular diseases is essential and relies on analyzing cardiac images acquired using medical imaging modalities. Cardiac Magnetic Resonance Imaging ( $\boldsymbol{C M R I})$ is generally known to be one of the most important diagnostic tools for the assessment of cardiac functions [4]. CMRI allows the acquisition of 3D and 4D (3D+time) cardiac image series, thus providing the means to perform a detailed analysis of global and local cardiac functions by enabling a quantitative assessment of functional parameters [5]. Among the cardiac chambers, the Left Ventricle $(\boldsymbol{L} \boldsymbol{V})$ is quite frequently analyzed because it provides $80 \%$ of the cardiac function. In fact, its main function is pumping oxygenated blood to the entire body to ensure any normal activity. Thus, ventricular segmentation often refers to $\boldsymbol{L} \boldsymbol{V}$ segmentation trough a manual outlining of the $\boldsymbol{L} \boldsymbol{V}$ myocardium. This approach represents the standard in the clinical routine despite of all these constraints in terms of the potential user error, considerable segmentation time and variability between observers [6].
Our goal is to faithfully delineate the myocardium through epicardial and endocardial contours' extraction. A good segmentation will be followed by a qualitative evaluation of the cardiovascular function such as measuring the left ventricular volume at end-systole and end-diastole. Since CMRI produces a large amount of data, processing these data becomes very time-consuming, a fastidious, and tedious task as well as an elaborate work. So, it is essential to speed up the extraction of the myocardium and make a full use of the pieces of information contained in these images [7].

Several approaches have been proposed for the LV segmentation. Most of them involve the computation of regional and global features, from which it is possible to detect cardiac dysfunction. Among these approaches, the deformable models algorithms and their variants have been widely used in cardiac MRI [8-10]. Nandagopalan and al. proposed a new approach combining the K-mean clustering algorithm and active contour model to detect cardiac borders [11]. Furthermore, active contour model based on Kurtosis wavelet energy is another promising segmentation technique. Since its first application on Synthetic Aperture Radar (SAR) images [12], this approach has attracted many researchers from different fields who used it for the segmentation of lesion structures in MRI images [13].

Another recent method based on sparse representation and dictionary learning has also been developed in the 
literature and it was applied on CINE and Cardiac Phaseresolved Blood oxygen level-Dependent (CP-BOLD) MR sequences [14]. An interesting algorithm applied to the SAR images [15] based on the combination of random subspace (RS), linear discriminating analysis, sparse regularization (LDASR) for feature space dimensionality reduction, supervised feature selection, and learning was used for multi-sensor data fusion based on multiple features. The main advantage of this technique is the invariance under intensity changes.

In addition to the segmentation methods described above, Convolutional Neural Network (CNN), is another important tool for the detection of $\mathrm{LV}$ contours. In the recent years, the $\mathrm{CNN}$ has revealed a good performance in image classification and segmentation in different fields, in particular, in SAR and MRI images [16,17]. Tan and al. [18] used this approach for the detection of endocardial contours in MRI images. Recently, CNN has been combined with other algorithms such as multilayer perceptron and deformable model to achieve better segmentation and classification performances [19,20]. Although CNNs algorithms demonstrated great results in different images segmentation applications, they still unable to segment cardiac structures in the small slices of the heart, especially in the apex.

It is important de mention that there are other approaches pursued to extract edges combining cellular automata and fuzzy rules [21] or local spectral histogram (LSH) [22] which are mainly applied on Synthetic Aperture Radar (SAR) images but that can be adapted to LV images' segmentation due to the high degree of accuracy in contour delineation and noise processing.

In latest works, an algorithm in [23] was proposed discriminate between objects of interest including a main step of shape-based feature extraction that can also be used for CMR images.

In this paper, we suggest to compare a set of existing methods [26-56] that handle $\boldsymbol{L} \boldsymbol{V}$ segmentation in a shortMRI axis, and to choose the best one that assumes extracting and computing the maximum set of parameters. Moreover, we present a full-process of $\boldsymbol{L} \boldsymbol{V}$ myocardium segmentation from 3D+time cine-MRI sequences based on a parallel watershed transformation [35]. We also define several parameters, their formulas, and a semi-algorithm for main ones such that End-Diastolic / End-Systolic / Stroke volume and Ejection Fraction. Finally, we test our approach on an initial data base composed of twenty patients to evaluate the accuracy of our work.

The rest of the paper is organized as follows: Section 2 sets up a comparative study of some existing LV segmentation methods. Section 3 advances the process of LV segmentation based on a parallel watershed transformation. The computation of cardiac function parameters is presented in section 4 . The obtained results are discussed in section 5. A summary of the realised work as well as possible extensions are introduced in section 6 .

\section{Left ventricular segmentation methods using Short-Axis MR image}

The quantification of global and local cardiac functional parameters requires a delineation of endocardial and epicardial contours of the $\boldsymbol{L} \boldsymbol{V}$ from cine short-axis (SA) MRI Figure 1 shows a full-size $\boldsymbol{S A}-\boldsymbol{C M R}$ image of the $\boldsymbol{L} \boldsymbol{V}$ and its different components. Therefore, many segmentation methods are discussed in the literature [7] to be used for the above-mentioned purpose. In this section, a synthetic study is applied to a set of works that handles the segmentation of the left ventricular and either the right endocardial ventricular and/or epicardial contours. A presentation of several heart functional parameters, useful for the diagnosis of cardiovascular diseases, is given as well at the lastpart of this section.

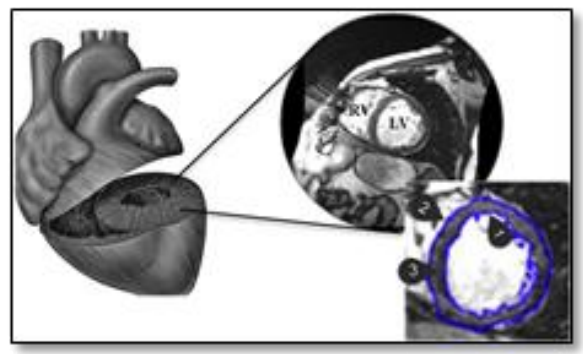

Fig. 1. A full size short-axis CMR image and a Region of Interest (ROI) identifying the heart: (1) Endocardium, (2) Epicardium and (3) Myocardium.

The accurate delineation of the endocardial and epicardial contours, with less or none user intervention, is a challenging task toward for the $\boldsymbol{L} \boldsymbol{V}$ segmentation in cardiac $\boldsymbol{C M R}$ Images. In fact, we mainly distinguish two categories to classify the existing $\boldsymbol{L} \boldsymbol{V}$ segmentation algorithms. Either the algorithm is based on no or a weak $(n \boldsymbol{w})$-prior or on a strong one.

\subsection{Left Ventricle segmentation based on nw-priors:}

Generally, $\boldsymbol{L} \boldsymbol{V}$ segmentation approaches based on (weak or no) prior knowledge are image driven. Thus, they are mainly based on image information and don't require any intensive training. According to the algorithm used for the segmentation process, these techniques can be classified as image-based methods, pixel classification, and deformable models.

a) Image-Based Methods: Endocardial and epicardial contours delineation leads to specific segmentation difficulties. Therefore, Image-based methods suggests to process them differently and separately following two steps:

- Step 1: The detection of endocardial contour, using either thresholding methods, and dynamic programming, or mathematical morphology operators.

- Step 2: The epicardium segmentation is performed by exploiting the endocardium boundary along with myocardial thickness or mathematical morphology operators.

b) Pixel classification: These methods are mostly used to deal with multiple images of the same scene especially in the case of multiple $\boldsymbol{M R I}$ or Multi-modality images. The main axis is to split images into different sections or classes having similar features. 
c) Deformable models: these methods use active contours or snakes and are based on deforming a curve iteratively, to minimize the energy consumption. Active contours have been widely used to segment medical images. Similarly to image-based methods, these approaches are based mainly on image information and don't require any intensive training as already mentioned.

\subsection{Left Ventricle segmentation based on strong priors:}

Automatic $\boldsymbol{L} \boldsymbol{V}$ segmentation is generally based on strong spatial priors such as statistical shape models or atlases. This is useful when the shape of the target region does not change significantly from one subject to another, which is a reasonable assumption for the $\boldsymbol{L} \boldsymbol{V}$. Such strong priors might reduce the intervention of the user's needs, but simultaneously enhance the cost of building a large training set manually. Methods belonging to this category are based on the models mentioned below:

a) Shape-driven deformable models: Those models develop an active curve/surface for the sake of minimizing the energy function and comprise a set of template shapes learned before. The evolution equation is computed by the minimization of functional energy, which contains a statistical shape constraint.

b) The Active Shape Model (ASM) / Active Appearance Model (AAM): According to these models, two steps have to be followed:

- Step 1: Building a statistical shape model following a Principal Component Analysis (PCA) of a set of aligned training shapes.

- Step 2: Finding segmentation in the current image by adapting the solution to the learned model then moving on to estimate various parameters such as translation, scaling or rotation.

c) Atlas-Based model: Using this model, the segmented image is obtained by mapping its coordinate space to that of an Atlas, often following a registration process. The main idea is to register the labeled Atlas onto the desired image to be segmented and then apply the obtained transformations onto the Atlas. The obtained results can then be propagated over time through the cardiac cycle following the same principle.

\subsection{Comparative study:}

Within this sub-section, we put forward a comparative study of segmentation methods that will be enhanced by synthesis tables, for each one of the above-cited categories. The classification will be strongly guided by the works presented in [7][24][25]. Based on their experimental conditions, segmentation methods belonging to the first and the second categories are synthesized respectively in Table $\boldsymbol{1}$ and 2. Notations and acronyms used in these two tables are explained below:

- Basic method principle: The segmentation method used to detect the ventricular contours.

- Author: The person who wrote and performed the algorithm.
- $\boldsymbol{L} \boldsymbol{V} / \boldsymbol{R} \boldsymbol{V}$ : indicate whether segmentation's results are provided on both LV (resp. RV) epicardial and endocardial contours ( $\mathrm{LV}, \mathrm{RV}$ ), or only on the endocardial (LVv, RVv).

- Magnetic Resonance Image (MRI): the acquisition type of cardiac $\boldsymbol{C M R I}$ series:
2D: 2 Dimensions
3D: 3 Dimensions
4D: 4 Dimensions (3D+ time)

- User Interaction (UI): might be used to initialize the segmentation. We distinguish three cases:

AHL: Automatic Heart Localization;

Use 1: The segmentation requires the selection of $\mathrm{LV}$ center-point or drawing a circle.

- Use 2: based on manually segmenting of the first image of the sequence.

- External Information (EI): External information combined during the segmentation process might be a weak prior.

- AM: Anatomic Model such as the circular aspect of the LV (Transformation into polar coordinates or use of radial lines); Simple spatial relationships ( $\mathrm{RV}$ is positioned on the left side of the LV); Use of a bullet shaped, cylinder or an ellipsoid volumetric model.

- SM: Statistic Model namely a set of manually drawn borders gathered and synthesized into a statistical model, might also be used.

- Methods making no use of external information are specified with a hyphen (-).

- Motion Information (MI): as the heart is a moving organ, its motion can be considered in the segmentation process.

- P: approaches that propagate an initial segmentation result on the whole cardiac cycle by repeating their algorithm on each image.

- M: approaches that explicitly take motion into account.

- Ventricular Function Evaluation (VFE): A qualitative performance of a segmentation method is quantified through the validation against a ground truth. The precision of the segmentation is also evaluated through quantitative measurements such as:

- $\quad$ S: Surface;

- $\quad \boldsymbol{E F}$ : Ejection Fraction;

- $\quad \boldsymbol{S V}:$ Stroke Volume;

- LVM: LV Mass;

- CO: Cardiac Output;

- WT: Wall Thickness;

- $\quad$ (-): if no clinical parameter is calculated;

Among the different presented algorithms, the one that meets our needs will be chosen according to qualitative and quantitative criteria based on each algorithm score. Table 3 represents the top five algorithms based on $n \boldsymbol{w}$-prior. Otherwise, the top three algorithms based on strong priors are listed in Table 4. 
Table 1 Synthesis of LV segmentation methods based on $\boldsymbol{n} \boldsymbol{w}$-prior

LV/RV: LEFT AND/OR Right VENTRICLE SEGMENTATION; MRI: MAGNETIC RESONANCE IMAGE; UI: UsER INTERACTION,

EI: EXTERNAL INFORMATION; MI: MOTION INFORMATION; VFE : VENTRICULAR FUNCTION EVALUATION.

\begin{tabular}{|c|c|c|c|c|c|c|c|c|c|}
\hline & Basic method principle & No. & Authors & LV/RV & MRI & UI & EI & MI & VFE \\
\hline \multirow{13}{*}{ 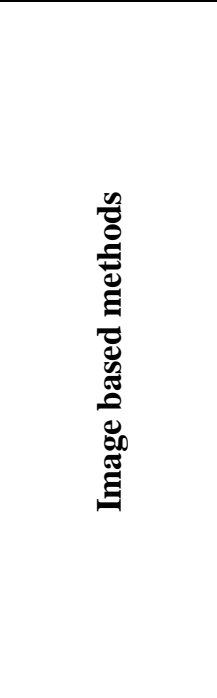 } & \multirow{4}{*}{ Thresholding } & 1 & Goshtasby and al [26] & $\mathrm{LVv}, \mathrm{RVv}$ & $2 \mathrm{D} / 3 \mathrm{D}$ & AHL & $\mathrm{AM}$ & - & $S$ \\
\hline & & 2 & Nachtomy and al [27] & LV & $2 \mathrm{D}$ & use 1 & $\mathrm{AM}$ & - & EF, SV \\
\hline & & 3 & Katouzian and al [28] & $\mathrm{LV}, \mathrm{RVv}$ & $2 \mathrm{D}$ & use 1 & - & - & $\mathrm{S}$ \\
\hline & & 4 & Lin and al [29] & $\mathrm{LVv}$ & $3 \mathrm{D}$ & AHL & $\mathrm{AM}$ & $P$ & - \\
\hline & \multirow{3}{*}{ Dynamic Programming } & 5 & Geiger and al. [30] & LV & $2 \mathrm{D}$ & use 2 & - & $\mathrm{P}$ & - \\
\hline & & 6 & Lalande and al. [31] & LV & $2 \mathrm{D}$ & use 1 & $\mathrm{AM}$ & - & SV \\
\hline & & 7 & Liu and al. [32] & $\mathrm{RVv}$ & $2 \mathrm{D}$ & use 2 & $\mathrm{AM}$ & - & - \\
\hline & Shortest Path Algorithms & 8 & Jolly and al [33] & LV & $4 \mathrm{D}$ & AHL & $\mathrm{AM}$ & $\mathrm{P}$ & $S$ \\
\hline & \multirow{2}{*}{$\begin{array}{l}\text { Mathematical Morphology / } \\
\text { Watershed transformation }\end{array}$} & 9 & Cousty and al [34] & LV & $3 \mathrm{D} / 4 \mathrm{D}$ & use 1 & $\mathrm{AM}$ & $\mathrm{M}$ & $\mathrm{EF}, \mathrm{LVM}$ \\
\hline & & 10 & Mahmoudi and al. [35] & LV & $3 \mathrm{D} / 4 \mathrm{D}$ & use 1 & $\mathrm{AM}$ & $\mathrm{M}$ & - \\
\hline & \multirow{2}{*}{ Gradient } & 11 & Lu and al [36] & LV & $3 \mathrm{D}$ & AHL & $\mathrm{AM}$ & $\mathrm{P}$ & $\mathrm{EF}, \mathrm{MM}, \mathrm{V}$ \\
\hline & & 12 & Waiter and al. [37] & LV & $3 \mathrm{D}$ & use 1 & - & - & $\mathrm{EF}$ \\
\hline & Graph Searching & 13 & Lee and al. [38] & LV & $3 \mathrm{D}$ & use 1 & $\mathrm{AM}$ & - & $\mathrm{EF}, \mathrm{V}, \mathrm{M}$ \\
\hline \multirow{4}{*}{$\begin{array}{c}\text { Pixel } \\
\text { Classification }\end{array}$} & KNN classifier & 14 & Hadhoud and al [39] & LV & $3 \mathrm{D}$ & AHL & - & - & - \\
\hline & Clustering & 15 & Cocosco and al. [40] & $L V v, R V v$ & $3 \mathrm{D}$ & AHL & $\mathrm{AM}$ & - & $\mathrm{EF}, \mathrm{V}$ \\
\hline & Neural Networks & 16 & Stalidis and al. [41] & LV & $2 \mathrm{D}$ & use 1 & - & M & - \\
\hline & Fuzzy KNN + Graph-Cut & 17 & Kedenburg and al. [42] & LV & $2 \mathrm{D}$ & AHL & $\mathrm{AM}$ & - & $\mathrm{V}$ \\
\hline \multirow{3}{*}{$\begin{array}{l}\text { Deformable } \\
\text { models }\end{array}$} & Shape Based Matching & 18 & Papademetris and al. [43] & LV & $3 \mathrm{D}$ & use 1 & SM & $\mathrm{P}$ & - \\
\hline & Active Contours & 19 & Santarelli and al. [44] & LV & $2 \mathrm{D}$ & use 2 & - & $\mathrm{P}$ & $\mathrm{V}, \mathrm{M}$ \\
\hline & 3D Active Contours & 20 & Heiberg and al. [45] & LV & $3 \mathrm{D}$ & use 1 & $\mathrm{AM}$ & $\mathrm{P}$ & $\mathrm{S}$ \\
\hline
\end{tabular}

Table 2 Synthesis of LV segmentation methods based on strong priors:

LV/RV: LEFT AND/OR Right VENTRICLE SEGMENTATION, MRI: MAGNETIC RESONANCE IMAGE, UI: USER INTERACTION,

EI: EXTERNAL INFORMATION; MI: MOTION INFORMATION; VFE: VENTRICULAR FUNCTION EVALUATION.

\begin{tabular}{|c|c|c|c|c|c|c|c|c|c|}
\hline & Basic method principle & No. & Authors & LV/LR & MRI & UI & EI & MI & VFE \\
\hline \multirow{4}{*}{$\begin{array}{c}\text { Shape-driven } \\
\text { Deformable } \\
\text { Model }\end{array}$} & Level-Sets + Stochastic & 1 & Lynch and al. [46] & LV & $2 \mathrm{D}$ & use 1 & SM & - & $\mathrm{S}$ \\
\hline & PCA + Bayesian Approach & 2 & Sun and al. [47] & LV & $2 \mathrm{D}$ & use 2 & SM & $\mathrm{M}$ & - \\
\hline & Level-Sets & 3 & Khalifa and al [48] & $\mathrm{LV}$ & $3 \mathrm{D}$ & AHL & SM & - & - \\
\hline & $\begin{array}{c}\text { Level-Sets + Deep Belief } \\
\text { Network }\end{array}$ & 4 & Ngo and al [49] & LV & $2 \mathrm{D}$ & use 1 & SM & M & - \\
\hline \multirow{4}{*}{$\begin{array}{c}\text { Active Shape / } \\
\text { Active Appearance } \\
\text { Models }\end{array}$} & $\mathrm{AAM}+\mathrm{ASM}$ & 5 & Zhang and al. [50] & LV, LRV & $4 \mathrm{D}$ & use 2 & SM & - & $\mathrm{V}, \mathrm{M}$ \\
\hline & Principal Component Analysis & 6 & Inamdar and al. [51] & LV & $3 \mathrm{D}$ & use 2 & SM & $\mathrm{M}$ & - \\
\hline & 2D + Time AAM & 7 & Lelieveldt and al. [52] & LV & $2 \mathrm{D}$ & AHL & SM & - & $\mathrm{V}, \mathrm{M}$ \\
\hline & 2D AAM + 3D ASM & 8 & Zambal and al. [53] & LV & $2 \mathrm{D} / 3 \mathrm{D}$ & - & SM & - & - \\
\hline \multirow{3}{*}{$\begin{array}{c}\text { Atlas } \\
\text { Based models }\end{array}$} & Anatomical Atlas + NRR & 9 & Lorenzo and al. [54] & LV, LRV & $4 \mathrm{D}$ & use 2 & SM & $P$ & $\mathrm{~V}$ \\
\hline & Probabilistic Atlas + NRR & 10 & Lötjönen and al. [55] & $\mathrm{LV}, \mathrm{LRV}$ & $3 \mathrm{D}$ & - & SM & - & - \\
\hline & Anatomical Atlas + NRR & 11 & Zhuang and al. [56] & LV, LRv & 4D & - & SM & - & $\mathrm{V}$ \\
\hline
\end{tabular}


The main criterion to choose the suitable segmentation algorithm, is to be simple, easy to implement and have the ability of (i) Delineating precisely the endocardial, epicardial and myocardial boundaries of left ventricle; (ii) Operating on $\mathbf{3 D}$ and $(\mathbf{3 D + t})$ cardiac MRI sequences; (iii) Offering the best performance in terms of execution time; and (iv) Computing the maximum number of parameters to assess the cardiac function.

Table 3: Top five algorithms of LV segmentation based $\boldsymbol{n} \boldsymbol{w}$ -

\begin{tabular}{c|l} 
prior. & \\
\hline 1 & Cousty and al (2010) \\
\hline 2 & Mahmoudi and al. (2017) \\
\hline 3 & Jolly and al (2009) \\
\hline 4 & Lu and al. (2013) \\
\hline 5 & Cocosco and al. (2008) \\
\hline
\end{tabular}

Table 4: Top three algorithms of LV segmentation based on strong priors.

\begin{tabular}{c|l}
\multicolumn{2}{c}{ strong priors. } \\
\hline 1 & Zhuang and al. (2008) \\
\hline 2 & Zhang and al. (2010) \\
\hline 3 & Lorenzo-Valdés and al. (2004) \\
\hline
\end{tabular}

According to the comparative study and the abovementioned criteria, the spatiotemporal watershed cuts method is selected considering the images spatial and temporal gradient.

In fact, this method allows producing spatial segmentations as well as temporal consistency between the successive 3D segmentation sequences obtained over time. A parallel topological watershed algorithm is introduced in [35] and it can be seen as an extension of the introduced watershed transformation presented in [34] that is suitable for shared memory parallel machines. It will be used on the LV segmentation process that will be developed in the next section.

In the remainder of this section, we will try to establish a link between the previous segmentation techniques announced and the in-depth learning technique, which is increasingly demonstrating its effectiveness in the analysis of cardiac MR images. This technique is mainly based on the use of 2D convolutional neural networks (CNN) and the analysis of MRI data, cut by cut.

In the remainder of this section, we will try to establish a link between the previous segmentation techniques announced and the in-depth learning technique, which is increasingly demonstrating its effectiveness in the analysis of cardiac MR images. This technique is mainly based on the use of 2D convolutional neural networks (CNN) and the analysis of MRI data, cut by cut.

Indeed, some studies describe an in-depth learning framework to extract the characteristics relevant to segmentation. For example, Kong and al. [57] developed a temporal regression framework to identify tele-diastolic and tele-systolic occurrences of the cardiac cycle by integrating a $2 \mathrm{D}$ CNN into a recurrent neural network (RNN). The CNN was used to code the spatial information, while the RNN was used to decode the temporal information. Other work,
Emad and al. [58], uses a CNN per patch to locate the VG in cardiac MRI sections. Finally, Zhang and al. [59] introduce a simple CNN to automatically detect missing sections (apical and basal) during cardiac examinations to evaluate the quality of MRI acquisitions.

The literature also describes other studies that use in-depth learning methods combined with conventional cardiac segmentation tools, such as Yang and al. [60] who have developed a combined $\mathrm{CNN}$ and multi-atlas approach to performing VG segmentation. In particular, a deep architecture has been formed to learn in-depth functionalities offering optimal performance for the label merging operation typically involved in multi-atlas segmentation. Ngo and al. [61] use a deep belief network $(\mathrm{DBN})$ to accurately initialize and guide a level-set model to segment the left ventricle.

Other techniques, introduced by Rupprecht and al. [62], integrate a "patch-based CNN" into a semi-automatic active contour (snake) to segment the contours of the heart. Alternatively, Avendi and al [20] propose an approach combining the deeplearning model and the deformable model to automatically segment the left ventricle of the heart. The introduced approach [20] launches a simple CNN that locates and cuts the VG before the presegmented shape is refined with a deformable model. It is important to note that there is an intermediate phase of pre-segmentation of the VG based on auto-encoders.

Finally, very few publications introduce only in-depth learning techniques to segment the ventricles of the heart. A commun architecture is the totally deep convolutional neural network architecture to segment both the right and left ventricles. The recurring network [63] is also a good example. Indeed, this fully convolutional network (RECR) learns image representations from the complete stack of 2D sections, while the derived architecture makes it possible to take advantage of spatial dependencies between the slices using internal memory units. For more precise image analysis, there is another super-image resolution approach, based on a residual convolutional neural network model [64], which proposes the reconstruction of high-resolution 3D volumes from stacks of 2D images.

Although they can provide specific results, in-depth learning methods face many limitations, mainly the large number of images they require during learning. Sharing this data often raises many legal issues related to the protection of patients' privacy. It is also important to note that there is no standard for automatic data learning algorithms without forgetting the fact that there is no way to correct their output in case of error. In addition, the formation of a system capable of treating healthy and pathological cases from cardiac MRI images remains an open question for all previously presented techniques due to the absence of large annotated data sets on pathological cases. 


\section{Left ventricular segmentation}

In this section, we present the principles and the different steps of $\boldsymbol{L} \boldsymbol{V}$ segmentation based on a parallel watershed transformation [35] before moving to considered algorithms for functional parameters computed in the next step. The Left Ventricular Myocardium ( $\boldsymbol{L V M}$ ), which is the object of interest, is surrounded by the epicardial $(\partial \boldsymbol{E} \boldsymbol{p})$ and the endocardial $(\boldsymbol{\partial E n})$ boundaries, as shown in Figure 2. These two borders form the left ventricular chamber $(\boldsymbol{L V C})$. The $\boldsymbol{L V C}$ myocardium $(\boldsymbol{L V C M})$ is delimited $(\boldsymbol{\partial E \boldsymbol { E n }})$ and surrounded by the left ventricular background ( $\boldsymbol{L V B})$ [39].

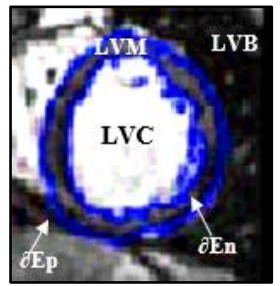

Fig. 2. Objects of interest in left ventricle image

\subsection{Automatic endocardial border detection:}

The segmentation of the endocardium depends on segmenting the $\boldsymbol{L} \boldsymbol{V}$ cavity $(\boldsymbol{L} \boldsymbol{V})$, by extracting different features such that gradient magnitude, largest eigenvalue, the output of the median filter and gray value. Figure 3 illustrates the different endocardial segmentation steps described below:

- First, the recognition process involves finding a marker for the $\boldsymbol{L} \boldsymbol{V} \boldsymbol{C}$ which is a subset made of points that certainly belong to the $\boldsymbol{L V C}$.

- Second, the delineation is performed by dilating this marker in a mask made of points that possibly belongs to the $\boldsymbol{L} \boldsymbol{V} \boldsymbol{C}$.

- Following the geometric properties of (2En), we do not apply any smoothing to the object obtained after delineation.

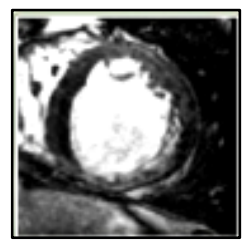

(a)

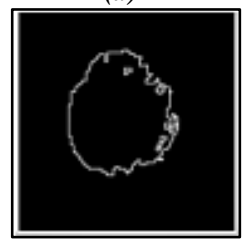

(c)

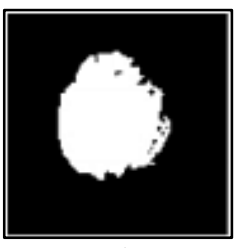

(b)

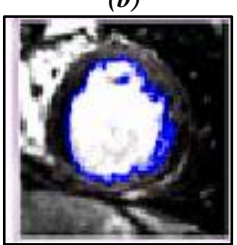

(d)
Fig. 3. Automatic endocardial border detection: (a) Original short-axis view image; (b) Result from edge detection; (c) Endocardial border after edge detection; (d) Final result.

\subsection{Automatic epicardial border detection:}

The LVCM segmentation is performed while preserving some $L V C$ anatomical constraints such that the left ventricular myocardium that does not have any hole. This leads to the following constraint: $E p B \cap E n B=\varnothing$.

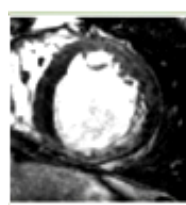

(a)

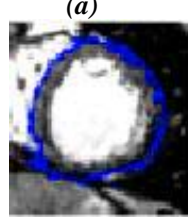

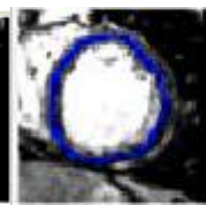

(b)

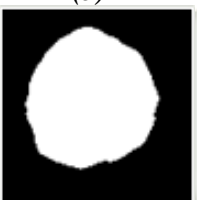

(e)

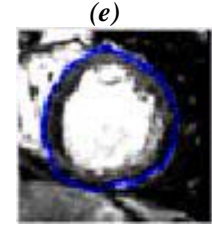

(g)

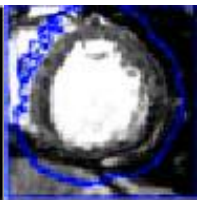

(c)

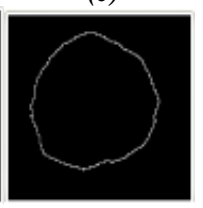

(f)
Fig. 4. Automatic epicardial border detection:

(a) Original short-axis image, (b) mLVCM selection,

(c) mLVB selection, (d) Result from PT-watershed algorithm, (e) Result from edge detection, $(f)$ Epicardial border after edge detection, (g) Final result.

The $\boldsymbol{L} \boldsymbol{V C M}$ segmentation process, as represented in Figure 4, is ensured by a Parallel Topological $(\boldsymbol{P T})$ watershed-based transformation [35] and performed following three steps:

- The recognition step consists in finding markers for both $\boldsymbol{L V} \boldsymbol{C M}$ and $\boldsymbol{L} \boldsymbol{V B}$ in order to separate them. Two markers named $\boldsymbol{m L} \boldsymbol{V} \boldsymbol{C M}$ and $\boldsymbol{m L} \boldsymbol{V B}$ are used respectively to mark $\boldsymbol{L} \boldsymbol{V C M}$ and $\boldsymbol{L} \boldsymbol{V B}$.

- Then, the delineation is performed by the PTwatershed algorithm using the above-selected markers as inputs.

- The segmentation is followed by a smoothing postprocessing based on the alternating sequential filter to regularize the $\boldsymbol{\partial E \boldsymbol { E }}$ and to restore a correct shape of the intensity information.

\subsection{Automatic myocardial border detection:}

The last step of the $\boldsymbol{L} \boldsymbol{V}$ segmentation process is to automatically detect the myocardial borders. The main task is joining two images obtained from Epi / Endo-cardium in previous steps. A special function is used to subtract an image from another one. The myocardial segmentation's steps, as well as results, are shown in Figure 5.

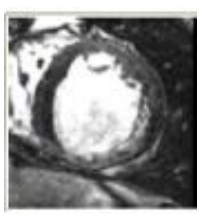

(a)

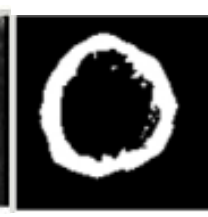

(b)

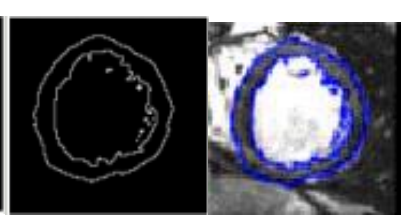

(c) (d)
Fig. 5. Automatic myocardial border detection:

(a) Original short-axis view image; (b) Result from edge detection; (c) Myocardial border after edge detection;

(d) Final result. 


\section{Heart function evaluation}

\subsection{Global parameters computing}

According to [65] and [66], the performance of the segmentation algorithm is evaluated following both quantitative and qualitative points of view. Towards a quantitative evaluation of the detected endocardial and epicardial contours during the end-diastole $(\boldsymbol{E D})$ and endsystole $(\boldsymbol{E S})$ phases of all slices, several measurements are assessed allowing global and regional analyses of cardiac function from $\boldsymbol{C M R}$ images. In this section, we introduce main clinical parameters that can be computed after accurate delineation of endocardial and epicardial contours of the $\boldsymbol{L} \boldsymbol{V}$.

\subsubsection{Left ventricular volumes:}

Left ventricular volume [67] is measured for each phase by multiplying the contour area, the sum of the slice thickness, and the slice gap. The end-diastolic volume $(\boldsymbol{E D V})$ is obtained after the R-wave from the first phase. The endsystolic volume $(E S V)$ is measured in the phase where the smallest endocardial volume appears. Formula (1) and algorithm (1) describe volume computation.

- $\quad \mathrm{V}$ is expressed in $\boldsymbol{m l}$.

$$
V=Z x \sum S_{i}
$$

- $\quad \mathrm{Z}$ is the slice thickness in $\mathbf{c m}$.

- $\quad S_{i}$ is the surface of the cavity on the slice $i,\left(\mathrm{~cm}^{2}\right)$.

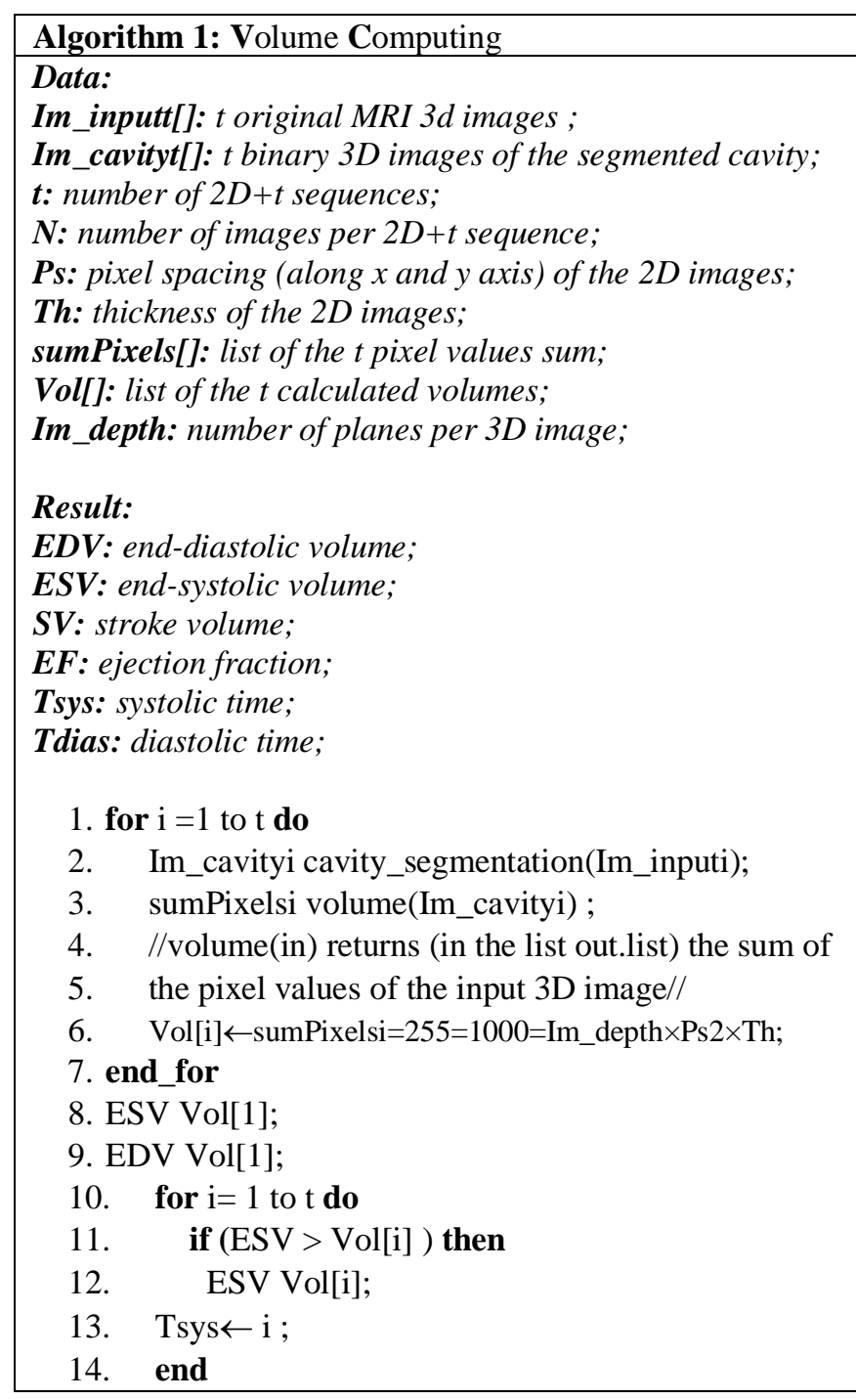

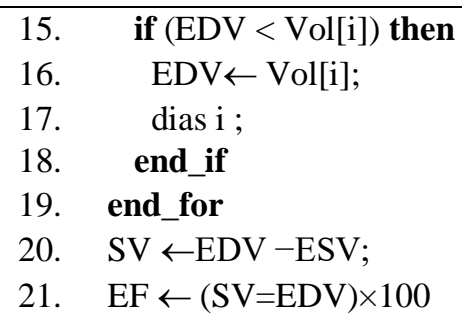

\subsubsection{Stroke Volume :}

The stroke volume $(\boldsymbol{S V})[\mathbf{6 8}]$ is simply defined as the difference between End-Diastolic Volume $(\boldsymbol{E D} \boldsymbol{V})$ and EndSystolic Volume $(\boldsymbol{E S V})$. It can be obtained by formula (2) and algorithm (2).

$$
S V=E D V-E S V
$$

\subsubsection{Ejection Fraction :}

The ejection fraction $(\boldsymbol{E F})$ is defined as the amount of blood ejected during the heart cycle. It is expressed as a fraction of the telediastolic volume and can be represented by the following equation (3). Different modalities for ejection fraction measurement are introduced in [69]. Authors introduce also the potential errors sources that can influence $\boldsymbol{F} \boldsymbol{E}$ computation.

$$
E F(\%)=\frac{V_{\text {endo }}\left(t_{D}\right)-V_{\text {endo }}\left(t_{S}\right)}{V_{\text {endo }}\left(t_{D}\right)} \times 100
$$

- $V_{\text {endo }}$ is the volume of the endocardium.

- $V_{\text {endo }}\left(t_{D}\right)=\max _{t}\left[V_{\text {endo }}(t)\right]$ is the end-diastolic volume

- $V_{\text {endo }}\left(t_{S}\right)=\min _{t}\left[V_{\text {endo }}(t)\right]$ is the end-systolic volume

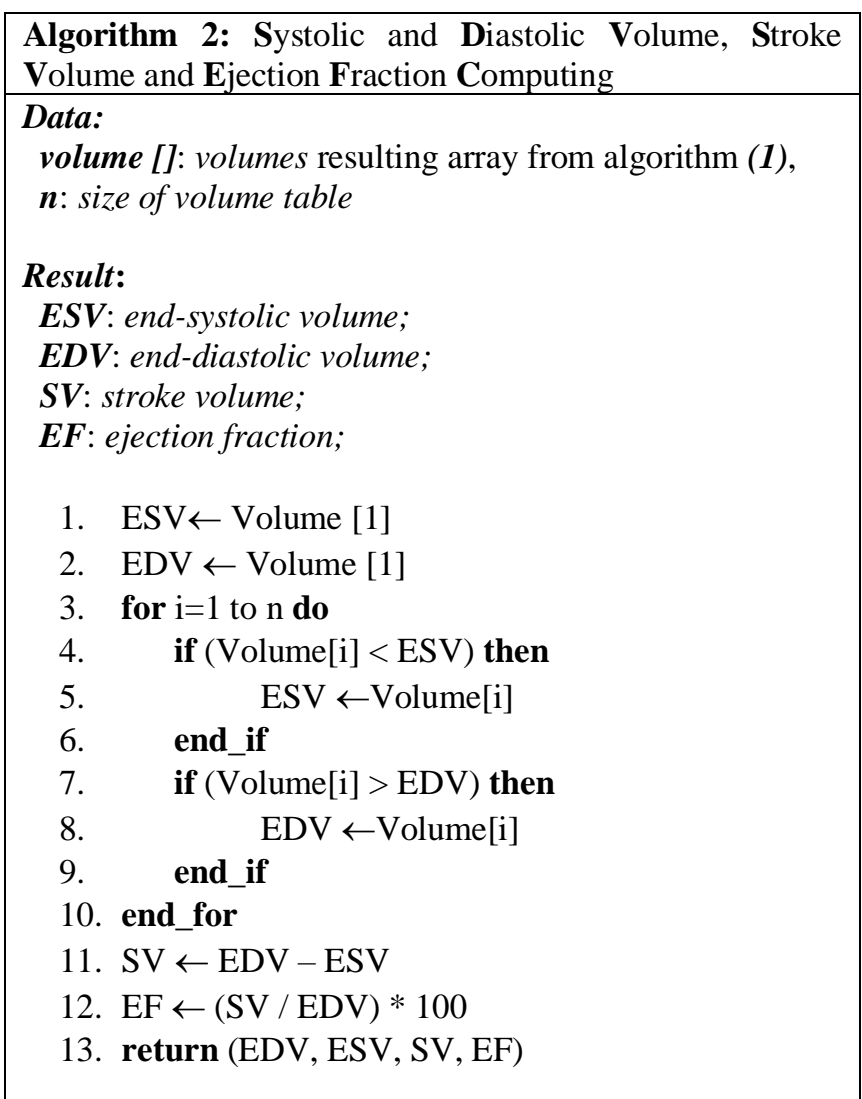




\subsubsection{Cardiac Output :}

Cardiac output $(\boldsymbol{C O})$ is defined by the amount of blood that heart pumps per unit of time. The unit used is $\mathbf{m L} / \mathbf{m i n}$. It is given by the following formula (4). A detailed review of the different techniques available for cardiac output measurement is presented in [70].

$$
C O=F_{\text {heart }} x(E D V-E S V)
$$

- $\quad F_{\text {heart }}$ is the cardiac frequency.

\subsubsection{Left ventricular mass:}

The left ventricular mass $(\boldsymbol{L} \boldsymbol{V M})[\mathbf{7 1}]$ is calculated in each phase by multiplying the left ventricular wall volume and the specific density of myocardium like shown by formula (5).

$$
\operatorname{LVM}(g)=\rho \times(E D V-E S V)
$$

- $\quad \rho$ is the density of the myocardium $(1.05 \mathrm{~g} / \mathrm{ml})$.

\subsubsection{Shortening Fractional:}

The shortening fractional $(\boldsymbol{S F})$ examines the shortening degree of the left ventricular diameter between the enddiastolic and the end-systolic. It is given by the following formula (6). The recommended calculations for $\mathrm{LV}$ function assessment [72], using changes in the LV dimensions and volumes between LV diastole and systole, are mainly $\boldsymbol{F S}$, $\boldsymbol{E F}, \boldsymbol{S} \boldsymbol{V}$ and $\boldsymbol{C O}$.

$S F(\%)=\frac{E D D-E S D}{E D D} \times 100$

- $\quad \boldsymbol{E D D}$ is the End-Diastole-Diameter.

- $\quad \boldsymbol{E S D}$ is the End-Systole-Diameter.

\subsubsection{Peak Ejection and Filling Rate:}

The peak ejection rate (PER) is defined as the maximum decrease of left ventricular volume per unit of time. The time to peak ejection rate (TPER) is defined as the time offset from the moment of $\boldsymbol{P E R}$ to the R-wave. Similarly, the peak filling rate $(\boldsymbol{P F R})$ is defined as the maximum increase of left ventricular volume per unit of time. The time to peak filling rate (TPFR) is defined as the time offset of the moment of $\boldsymbol{P F R}$ from the moment of end-systole. Both $\boldsymbol{P E R}$ and PFR were expressed in $\boldsymbol{E D V / s}$ [73][74]. Unfortunately, at this stage, we have not found in the literature a mathematical formulation to deduce or calculate these two parameters.
Algorithm 3: Wall Thickness Computing

Data:

Im_i: image of the binarized myocardial wall;

PS: pixel spacing;

$\boldsymbol{n}$ : number of the images;

Tsys: systolic time

Tdias: diastolic time

Th: Im_i thickness (z_voxel size)

xs: Im_i width ( $x \_$voxel size)

ys: Im_i height (y_voxel size)

Result:

SWT: systolic wall thickness;

DWT: diastolic wall thickness;

WT: percent wall thickening;

1. Im_sys $\leftarrow$ extractpale(im_Tsys, Th,xy) //extract the basal plane 2D xy at Tsystole time//

2. d __sys $\leftarrow$ crop $\left(\operatorname{Im} \_s y s, 0, x s / 2, y s / 2,1\right)$ // extracts the rectangle with upper left corner (0xs), of width ys/2 and height 1 from the input image Im_sys//

3. Im_dyas $\leftarrow$ extractpale $\left(i m \_T d y a s, T h, x y\right)$ //extract the basal plane 2D xy at Tsystole time//

4. d _dyas $\leftarrow$ crop $\left(\operatorname{Im} \_d y a s, 0, x s / 2, y s / 2,1\right)$

// extracts the rectangle with upper left corner (0xs), of width ys/2 and height 1 from the input image Im_dyas//

5. SWT $\leftarrow$ volume $\left(\right.$ d_sys $_{-} / 255 *$ Ps

6. DWT $\leftarrow$ volume $\left(d_{\text {d_dyas }}\right) / 255^{*}$ Ps $/ /$ volume calculates the sum of pixels that's why we divide by $255 / /$

7. $\mathrm{WT}=((\mathrm{SWT}-\mathrm{DWT}) / \mathrm{DWT}) * 100$

8. return $(\mathrm{SWT}, \mathrm{DWT}, \mathrm{WT})$

\subsubsection{Wall Thickness:}

The wall thickness (WT) is defined by the distance between the endocardial and epicardial contours. This is usually obtained by a method called "Center-line" which consists in computing the distance between the two edges along the normal line to the surface of the two contours. The percentage of wall thickening [75] (WTP) is obtained from the end-systolic wall thickness (ESWT) and the wall thickness of end-diastolic (EDWT) as given by formula (7) and algorithm (3).

$W T P(\%)=\left[\frac{(E S T-E D T)}{E D T}\right] x 100$ 


\section{Results and Discussion}

In this section, we start by presenting a qualitative and quantitative validation of left ventricular segmentation based on a parallel watershed transformation [35].

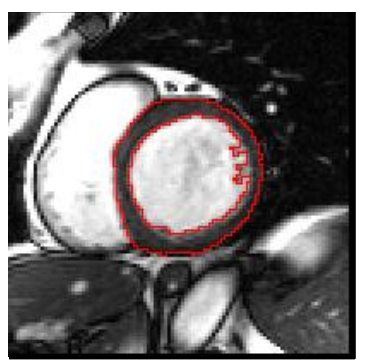

(a)

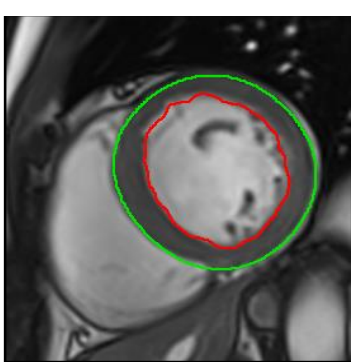

(b)
Fig. 6. End diastolic cine-MRI images illustrating the results of automatic myocardial contours detection using (a) cardiac MRI analysis software and (b) parallel watershed transformation.

In order to test the accuracy of the proposed algorithm for the segmentation of myocardial borders, a commercially available cardiac MRI analysis software (CVi42, version 5.5.1, Circle Cardiovascular Imaging Inc., Calgary, Canada) was used to automatically delineate the endocardial and epicardial contours. An expert radiologist (9 years of CMRI experience) reviewed the segmentation protocol and validated the results. If needed, a manual contour adjustment was applied: Myocardial contours were segmented from base to apex using 20 data sequences in a short axis view. The papillary muscles were excluded from the left ventricular mass. Figure $\mathbf{6}$ illustrates an example of myocardial contours' delineation using two segmentation methods, applied on cine MRI image at a diastolic instant. The results show an agreement between the two algorithms' performances.

In addition, the visual inspection of myocardial segmentation shows that the proposed algorithm is more accurate in segmenting myocardial borders in regions where the different structure intensities are very similar. Another observation is the ability of myocardial segmentation algorithm that is based on watershed transformation [35] to correctly delineate the endocardial contour with the presence of trabeculae and papillary muscles. This qualitative assessment is also confirmed if we compare the segmentation results of the second approach, see figure $\mathbf{7}$, for two patients considered pathological and healthy respectively. Cuts are extracted from three zones: basal, intermediate and apex. They show suitably identifiable boundaries of the myocardium.

As the cross-sections used by the two approaches were not the same, it proved very difficult to apply a quantitative comparison between the two resulting images, show in figure 6, using Mean Absolute Distance (MAD), Hausdorff Distance (HD) or Dice Coefficient (DC) [76]. To overcome this obstacle and maintain a quantitative assessment, we move on to the assessment of the calculated parameters.

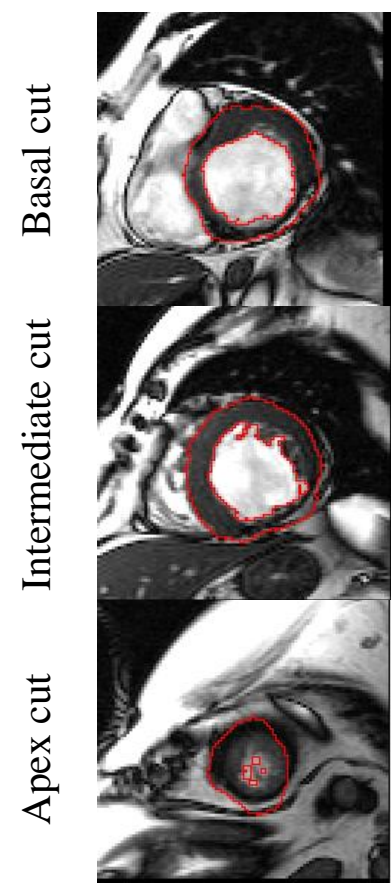

(a)

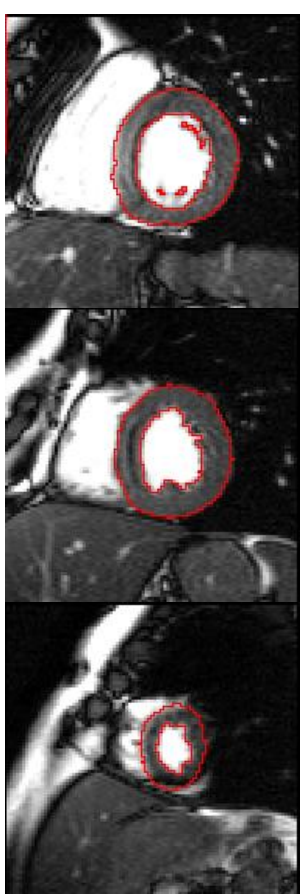

(b)
Fig. 7. T diastolic cine-MRI images illustrating the results of automatic myocardial contours detection using parallel watershed transformation for $(\boldsymbol{a})$ Patient considered pathological and $(\boldsymbol{b})$ Patient considered healthy

Indeed, several global LV parameters including enddiastolic volume, end-systolic volume, stroke volume, ejection fraction, and myocardial mass were measured using the same software. Measurements were obtained using 20 sequences of patient's MR images (20-25 images per sequence) gathered from University Hospital Fattouma Bourguiba Monastir. The image which corresponds to the end diastolic was selected as the first image of the series while the end systolic image was chosen as the smallest LV volume.

As an additional quantitative assessment, the clinical measurements obtained from the proposed approach, see table 5, were compared to those derived from the automated segmentation, see table 6, using Cardiac MRI analysis software. The values that are most relevant in Table 5 are those of the WT. Indeed, for a healthy patient, the myocardium thickens during the systole. On the other hand, for a pathological patient, the myocardium shows a decrease in this thickening or even a thinning. Thus the percentage of thinning must be positive (above 17\%) for a healthy patient. For pathological cases with acute cardiomyopathy, zero or even negative percentages can be obtained (parietal thinning in systole). The percentage $0 \%$ for patients (P1, P11, P18) indicates that the parietal thickness is the same in systole and diastole, so the myocardium is abnormal. For the evaluation of other parameters, a Bland-Altman analysis was used to evaluate the degree of agreement between Left ventricular volumes and ejection fraction measurements derived from left ventricular segmentation based on a parallel watershed transformation and those from the automated segmentation. All statistical analyses were performed using IBM-SPSS Statistics (Windows, version 21.0). 
Table 5. Experimental results using PT-Watershed segmentation.

\begin{tabular}{llccllllll}
\hline & EDV & ESV & EF & CO & SV & LVM & SWT & DWT & WT \\
\hline P1 & 153,5 & 85 & 44,6 & 3,63 & 68,5 & 143,9 & 17.71 & 17.7 & 0 \\
P2 & 85,6 & 29 & 66,1 & 3,42 & 56,6 & 189,3 & 8.85 & 15.9 & 80 \\
P3 & 146,3 & 59 & 59,6 & 5,40 & 87,3 & 115,4 & 5.47 & 8.25 & 12 \\
P4 & 115 & 61 & 46,9 & 3,40 & 54 & 131,9 & 10.62 & 17.7 & 67 \\
P5 & 188,8 & 83 & 56,0 & 6,82 & 105, & 156,1 & 15.04 & 24.4 & 62 \\
P6 & 157,3 & 85 & 45,9 & 4,41 & 72,3 & 98,1 & 13.86 & 19.8 & 43 \\
P7 & 290 & 231, & 20,3 & 5,23 & 58,8 & 147,1 & 8.85 & 10.6 & 20 \\
P8 & 183,3 & 130 & 29,1 & 4,37 & 53,3 & 115,3 & 10.62 & 17.7 & 67 \\
P9 & 119 & 38,1 & 67,9 & 5,01 & 80,9 & 145,4 & 8.85 & 14.1 & 60 \\
P10 & 236 & 160, & 31,8 & 5,63 & 75,1 & 106,4 & 11.60 & 14.5 & 25 \\
P11 & 101,4 & 44,5 & 56,1 & 3,12 & 56,9 & 122,2 & 8.85 & 8.85 & 0 \\
P12 & 217 & 100 & 53,9 & 7,01 & 117 & 143,3 & 8.85 & 10.6 & 20 \\
P13 & 187,5 & 93,4 & 50,1 & 5,80 & 94,1 & 56,2 & 11.88 & 15.8 & 33 \\
P14 & 156,2 & 59,1 & 62,1 & 8,91 & 97,1 & 78,6 & 8.85 & 12.4 & 40 \\
P15 & 187 & 87,6 & 53,1 & 6,84 & 99,4 & 76,1 & 10.62 & 15.9 & 50 \\
P16 & 197 & 127, & 35,2 & 5,60 & 69,3 & 130,7 & 15.94 & 21.2 & 33 \\
P17 & 189 & 128 & 32,3 & 4,45 & 61 & 115,0 & 15.94 & 21.2 & 33 \\
P18 & 155 & 63,9 & 58,7 & 5,60 & 91,1 & 59,1 & 8.30 & 8.30 & 0 \\
P19 & 130,8 & 58,4 & 55,3 & 4,91 & 72,4 & 111,3 & 11.20 & 12.4 & 18 \\
P20 & 173,9 & 96 & 44,8 & 5,53 & 77,9 & 106,5 & 30.35 & 9.60 & 20 \\
\hline
\end{tabular}

The results of bland Altman analysis for left ventricular ejection fraction measurements, as shown in table 7 and figure 8 , reveal that the mean difference is very close to zero, which demonstrates that the myocardial contours segmentation based on watershed transformation gives unbiased automated measurement of global parameters. Also, the obtained $\boldsymbol{L} \boldsymbol{V E F}$ values are in line with those derived from literature. In addition, the $\boldsymbol{L} \boldsymbol{V}$ volumes measurements derived from the watershed transformation algorithm are in agreement with those derived from cardiac MRI analysis software with a little discrepancy between the $\boldsymbol{E D V}$ values obtained by the two methods.

Table 7. The results of the comparison between the two segmentation approaches using mean and standard deviation.

$*=$ calculated as mean \pm standard deviation $(S D)$.

$\operatorname{App}(\mathbf{1})=$ Automated myocardial segmentation

$A p p(2)=\mathrm{LV}$ segmentation based on parallel watershed transformation

\begin{tabular}{|c|c|c|c|}
\hline & $\boldsymbol{E D V} *(\boldsymbol{m l})$ & $\boldsymbol{E S V} *(\boldsymbol{m l})$ & $\boldsymbol{E F} *(\%)$ \\
\hline App(1) & $170.23 \pm 47$ & $91.94 \pm 45$ & $48.433 \pm 12$ \\
\hline App(2) & $168.97 \pm 88$ & $91.21 \pm 46$ & $48.70 \pm 13.4$ \\
\hline $\begin{array}{c}\text { Mean } \\
\text { Differences }\end{array}$ & 1.441 & 0.70 & -0.271 \\
\hline
\end{tabular}

For the latter values, we will study the homogeneity of variances for both approaches when computing $\boldsymbol{E D V}$. We use the Hartley test [77][78] since the number of patients is the same. This is essential to ensure that if the variation factor used in the experiment influenced the data, it did induce a change in mean, but not in variance. We consider the following two hypotheses:

Ho: $_{0}$ The variances of the samples are homogeneous

H1: At least one of the variances is significantly different from the others
Table 6. Experimental results using Cardiac MRI analysis software

\begin{tabular}{lllllll}
\hline & EDV & ESV & EF & CO & SV & LVM \\
\hline P1 & 154,97 & 85,66 & 44,66 & 3,67 & 69.3 & 144 \\
P2 & 88,1 & 30,2 & 66 & 3,5 & 57.9 & 189 \\
P3 & 148,67 & 58,68 & 60 & 5,57 & 99 & 116 \\
P4 & 117 & 62 & 47 & 3,46 & 55 & 132 \\
P5 & 191,2 & 84,1 & 56 & 6,9 & 107.1 & 156 \\
P6 & 159,6 & 86,3 & 45,9 & 4,47 & 73.3 & 98 \\
P7 & 292,4 & 232,8 & 20,4 & 5,3 & 59.6 & 148 \\
P8 & 185,5 & 131,7 & 29 & 4,41 & 53.8 & 115 \\
P9 & 120,7 & 38,9 & 67,8 & 5,07 & 81,8 & 145 \\
P10 & 238 & 162 & 32 & 5,7 & 76 & 107 \\
P11 & 103,82 & 45,42 & 56 & 3,2 & 47.82 & 122 \\
P12 & 217,83 & 100,6 & 53,8 & 7,02 & 117,2 & 143 \\
P13 & 189 & 94,5 & 50 & 5,82 & 91 & 56 \\
P14 & 157 & 60 & 61,7 & 8,9 & 81,8 & 78 \\
P15 & 188,7 & 88,4 & 53,1 & 6,9 & 100,3 & 76 \\
P16 & 198,24 & 129 & 35 & 5,6 & 69.24 & 130 \\
P17 & 189,5 & 128,32 & 32 & 4,46 & 61.18 & 114 \\
P18 & 156,4 & 64,6 & 58,7 & 5,64 & 91,8 & 59 \\
P 19 & 133 & 58,9 & 55,7 & 5,03 & 74.1 & 112 \\
P20 & 175,1 & 96,9 & 44,6 & 5,55 & 78,2 & 106 \\
\hline
\end{tabular}

$F_{\max }=\frac{s_{\max }^{2}}{s_{\min }^{2}}=1,87$

According to table 7, the standard deviation when computing $\boldsymbol{E} \boldsymbol{D} \boldsymbol{V}$ values is $\mathbf{8 8}$ using the LV segmentation based on parallel watershed transformation, while it does not exceed $\mathbf{4 7}$ for the automated myocardial segmentation approach. Thus, we can calculate the ratio between maximum and minimum variances of the two samples as shown in formula $(8)$.

In this study, we treat two populations $(k=2)$. The number of patients per population is twenty, so the degree of freedom is 19. With a 95\% confidence level, the Hartley table shows an $F_{t a b}$ value greater than 2.46 .

So we can conclude that $\left(F_{\max } \leq F_{t a b}\right)$, which makes the $\mathbf{H}_{0}$ hypothesis correct; it means that the variances have values close enough to each other that we accept the hypothesis that they are all homogeneous.

We also propose a second left ventricular volumes evaluation. The latter will be based on the median and interquartile range instead of mean and standard deviation. As shown in table 8, it maintains the correlation previously introduced between the results of the two approaches even if the disparity between measurements is large. The same mean differences mentioned above are maintained in all measurements. 
Table 8. The results of the comparison between the two segmentation approaches using median and interquartile range.

* $=$ calculated as median $\left[25^{\text {th }}-75^{\text {th }}\right.$ percentile $]$.

$\operatorname{App}(\mathbf{1})=$ Automated myocardial segmentation

$\boldsymbol{A p p}(\mathbf{2})=$ LV segmentation based on parallel watershed transformation

\begin{tabular}{|c|c|c|c|}
\hline & EDV *(ml) & ESV *(ml) & EF $^{*}(\boldsymbol{\%})$ \\
\hline $\boldsymbol{A p p}(\mathbf{1})$ & $165.67[134.6-188.9]$ & $85.0[59-120.2]$ & $51.67[32.5-58.1]$ \\
\hline $\boldsymbol{A p p}(\mathbf{2})$ & $167.35[136.9-190.7]$ & $85.9[59.1-121.3]$ & $51.55[37.4-58.0]$ \\
\hline
\end{tabular}

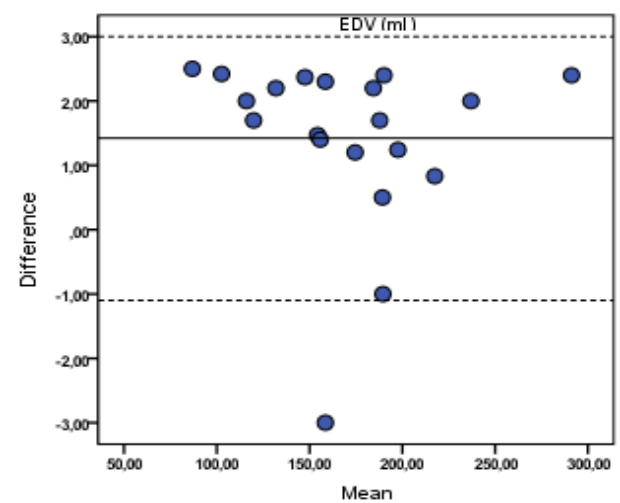

(a)

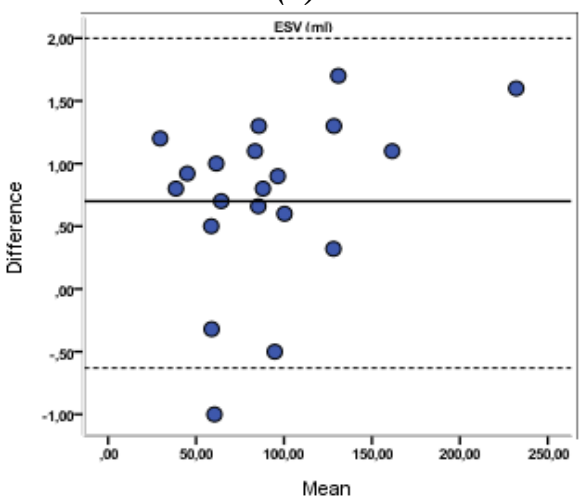

(b)

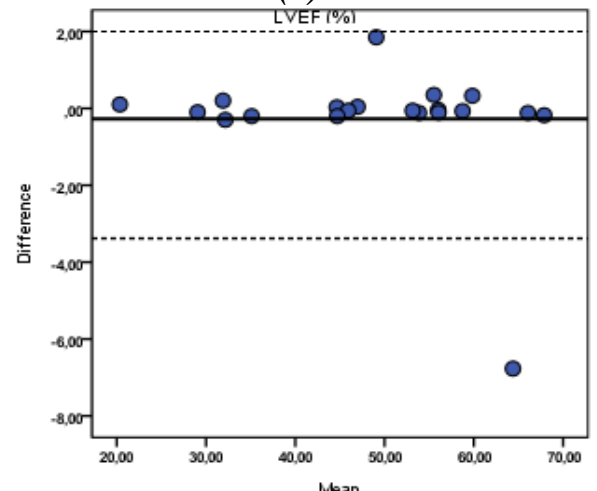

(c)

Fig.8 . The bland-Altman plots of the clinical measurements: (a)EDV values; (b) ESV values and (c)

LVEF (solid lines indicatemean differences; dashed lines indicate limits of agreement).
Furthermore, to demonstrate the clinical application of our algorithm, we investigated the association between global parameters computed from the proposed algorithm and a regional index of $\boldsymbol{L} \boldsymbol{V}$ contractility derived from [79]. The regional parameter used in this study is the amplitude of contraction. For more details about the regional feature computation, the reader should be referred to [79]. Linear regression analysis was performed to evaluate the correlation between $\boldsymbol{L V E \boldsymbol { E }}$ measurements and regional index of $\boldsymbol{L} \boldsymbol{V}$ contractility for the same 20 patients.

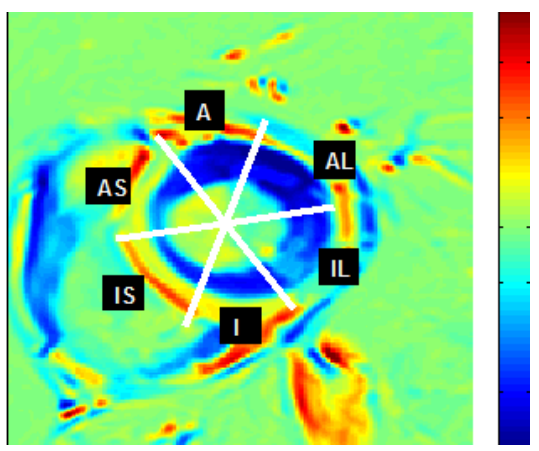

Fig. 9 Regional assessment of $\boldsymbol{L} \boldsymbol{V}$ dysfunction using parametric images for patient (P16) with myocardial infarction at the septal wall.

Figure 9 shows an example of regional assessment of cardiac wall motion abnormalities using parametric imaging for patient with myocardial infarction at the inferoseptal and anteroseptal walls $(\boldsymbol{P 1 6})$. The global parameters measurements computed after myocardial contours segmentation based on watershed transformation applied on the same patient's data, reveal a reduced $\boldsymbol{L} \boldsymbol{V}$ ejection fraction with a value of $35.2 \%$. The corresponding parametric image shows a decrease of amplitude contraction at the anteroseptal and inferoseptal walls indicating the presence of $\boldsymbol{L} \boldsymbol{V}$ dysfunction which is consistent with $\boldsymbol{L} \boldsymbol{V} \boldsymbol{E F}$ measurements.

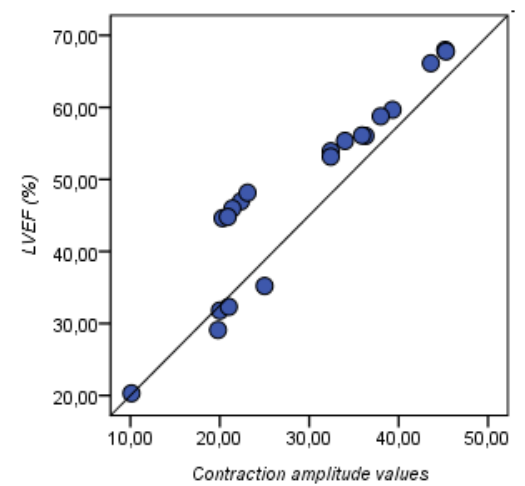

Fig. 10. The regression analysis plots illustrating the correlation between $\boldsymbol{L V E F}$ measurements derived from the watershed transformation algorithm and the regional index of $\boldsymbol{L} \boldsymbol{V}$ contractility from parametric images. 
The regression analysis shows a good correlation between $\boldsymbol{L} \boldsymbol{V E F}$ measurements derived from the watershed transformation algorithm and the regional index of $\boldsymbol{L} \boldsymbol{V}$ contractility from parametric images with a Pearson's correlation coefficient $\boldsymbol{r}=\mathbf{0 . 9 8 3}$ (for $\boldsymbol{p}<\mathbf{0 . 0 1}$ ). The diagram of regression analysis is shown in figure $\mathbf{1 0}$.

Previous experimental results demonstrate that the distinguished segmentation approach allows not only the computation of the highest number of parameters but also provides a satisfying measurement as close as conceivable to the actual values in accordance with normal values of various global and regional functional parameters.

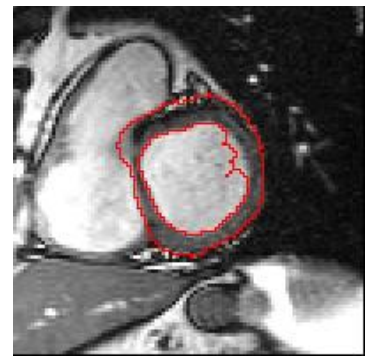

Fig. 11 Miss-segmented basal cut: Endocardial border poorly detected

It is important to note that our segmentation approach may face major difficulties, see figure 11, if the 2D cutting series suffers from a high signal-to-noise ratio. Indeed, the presence of noise will disorient the LPE algorithm even though watershed transformation approach is using twoselected markers as inputs:

- To extract the first marker, LV endocardium is dilated considering LV morphological prior (the thickness of the myocardium cannot be zero).

- The second marker is obtained by applying a second dilation considering the following prior: "the thickness of the left ventricular myocardium cannot exceed a certain threshold".

Watershed transform is strongly sensitive to image noise. Therefore, we use markers to reduce its sensitivity, but we cannot eliminate it.

\section{Conclusion and perspectives}

The present work introduces an advanced approach to assessing cardiac function. The first step is to extract the myocardium through left ventricular segmentation based on parallel watershed transformation. Thanks to a good segmentation quality, a second step is launched to estimate the global and regional parameters of the left ventricle in $3 \mathrm{D}+\mathrm{t}$ cine-MRI sequences. More than six clinical parameters, such that (End-Diastolic Volume $(\boldsymbol{E D V})$, EndSystolic Volume $(\boldsymbol{E} \boldsymbol{S} \boldsymbol{V})$, Stroke volume $(\boldsymbol{S} \boldsymbol{V})$, Ejection Fraction $(\boldsymbol{E F})$, Left Ventricular Mass $(\boldsymbol{L} \boldsymbol{V M})$ and Cardiac Output $(\boldsymbol{C} \boldsymbol{O})$ ), associated to the left ventricle function, are extracted with optimal computation time. The obtained results were compared to those returned by the Circle Cardiovascular Imaging tool. Degree of agreement between left ventricular volumes and ejection fraction measurements was evaluated using IBM-SPSS Statistics. An investigation on the association between global parameters computed from the proposed algorithm and a regional index of LV contractility has been also introduced. All results demonstrate the efficiency of our segmentation method for accurate myocardium detection and consequently enhance $\boldsymbol{L} \boldsymbol{V}$ functional quantification.

Nevertheless, there is much to do; future work [80] might include the support of right ventricular $(\boldsymbol{R} \boldsymbol{V})$ segmentation and extraction of associated parameters. However, many challenges can affect the performance of the right ventricular segmentation. First, there are qualitative problems with cardiac MRI images due to the low contrast intensity of the right heart portion, which makes it difficult to accurately determine if a pixel belongs to the hollow area of the $\boldsymbol{R} \boldsymbol{V}$, its outlines or its background. In addition, $\boldsymbol{R} \boldsymbol{V}$ segmentation is influenced by its morphology and structure in the cardiac cycle (from baseline to apex, from systole to diastole), and the enormous variation between acquired series from one patient to another, whether he is a healthy patient or is subject to a pathology.

\section{References}

[1] Ponikowski, P., Anker, S.D., AlHabib, K.F., Cowie, M.R., Force, T.L., Hu, S., Jaarsma, T., Krum, H., Rastogi, V., Rohde, L.E., Samal, C.S., Shimokawa, H., Siswanto, B.B., Sliwa, K., and Filippatos, G.: 'Heart failure: preventing diseaseand death worldwide', ESC Heart Fail,2014, 1 (1), pp 4-25.

[2] McMurray, J.J., Adamopoulos, S., Anker, S.D., Auricchio, A., Böhm, M., Dickstein, K.,Falk, V., Filippatos, G., Fonseca, C., Gomez-Sanchez, M.A., and al. "ESC guidelines for the diagnosis and treatment of acute and chronic heart failure", Eur. J. Heart Fail,2012, 14 (8), pp 803-869.

[3] Lozano, R., Naghavi, M, Foreman K., and al. "Global and regional mortality from 235 causes of death for 20 age groups in 1990 and 2010: a systematic analysis for the Global Burden of Disease Study 2010”. Lancet, 2012, 380, (9859), pp2095-128.

[4] Ripley, D.P., Musa, T.A., Dobson, L.E., and al.: "Cardiovascular magnetic resonance imaging: what the general cardiologist should know”, Heart, 2016,102,(19), pp1589-1603.

[5] Shah, S., Chryssos, E.D., Parker, H.: "Magnetic resonance imaging: a wealth of cardiovascular information”, Ochsner Journal, 2009, 9, (4):pp 266-77.

[6] Frangi, A.F., Niessen, W.J., Viergever, M.A.: "Threedimensional modeling forfunctional analysis of cardiac images: a review", IEEE Trans. Med. Imaging, 2001, 20, (1), pp 2-5.

[7] Petitjean C., and Dacher, J. N.: "A review of segmentation methods in short axis cardiac MR images", Med. Image Anal., Apr. 2011, 15, (2), pp. 169-184.

[8] Li, C., Huang, R., Ding, Z., Gatenby, JC., Metaxas, DN., Gore, JC.: "A Level Set Method for Image Segmentation in the Presence of Intensity Inhomogeneities With Application to MRI", IEEE Transactions on Image Processing July 2011, 20(7),pp.2007-2016. 
[9] Yang, C., Wu, W., Su, Y., Zhang, S. : « Left ventricle segmentation via two-layer level sets with circular shape constraint”, Magnetic Resonance Imaging May 2017, 38,pp. 202213.

[10] Constantinides, C., Chenoune, Y., Mousseaux, E., Roullot, E., Frouin, F.: "Automated heart localization for the segmentation of the ventricular cavities on cine magnetic resonance images", Computing in Cardiology 2010, pp.911-914 .

[11] Nandagopalan, S., Adiga, B.S., Dhanalakshmi, C., Deepak, N.. : "Automatic Segmentation and Ventricular Border Detection of 2D Echocardiographic Images Combining K-Means Clustering and Active Contour Model", Second International Conference on Computer and Network Technology 2010, Bangkok, Thailand.

[12] Akbarizadeh, G. "A New Statistical-Based Kurtosis Wavelet Energy Feature for Texture Recognition of SAR Images", IEEE Transactions on Geoscience and Remote Sensing, November 2012, 50, (11), pp. 4358-4368.

[13] Karthik, R., Menaka, R. :"Statistical characterization of ischemic stroke lesions from MRI using discrete wavelet transformation", Conference on Signal and Image Processing Application 2011 , pp. 384-389,

[14] Oksuz, I., Mukhopadhyay, A., Dharmakumar, R., Tsaftaris ,SA.: "Unsupervised Myocardial Segmentation for Cardiac BOLD. IEEE Trans Med Imaging". Nov 2017, 36(11), pp.2228-2238.

[15] Karimi, D., Akbarizadeh, G., Rangzan, K. and Kabolizadeh, M.: "Effective supervised multiple-feature learning for fused radar and optical data classification", in IET Radar, Sonar \& Navigation, May 2017., 11, (5), pp. 768-777.

[16] Yang, X., Song, Q., Su, Y.. : “Automatic segmentation of left ventricle cavity from short-axis cardiac magnetic resonance images". Med Biol Eng Comput. Sep 2017,55(9),pp.1563-1577.

[17] Li, Y., Peng, C., Chen, Y., Jiao, L., Zhou, L., Shang, R..: “A Deep Learning Method for Change Detection in Synthetic Aperture Radar Images", IEEE Transactions on Geoscience and Remote Sensing August 2019,57(8) ,pp. 5751-5763.

[18] Tan, LK., Liew, Y.M., Lim, E., McLaughlin, RA.: "Convolutional neural network regression for short-axis left ventricle segmentation in cardiac cine MR sequences"., Med Image Anal. Jul 2017,39;pp.78-86.

[19] Sharifzadeh, F., Akbarizadeh, G., Seifi Kavian, Y.: “Ship Classification in SAR Images Using a New Hybrid CNN-MLP Classifier", Journal of the Indian Society of Remote Sensing 2019, 47(4), pp 551-562.

[20] Avendi, M.R., Kheradvar, A., Jafarkhani, H. : “A Combined Deep-Learning and Deformable-Model Approach to Fully Automatic Segmentation of the Left Ventricle in Cardiac MRI", Journal of Medical Image Analysis, 2016, (30), pp 108-119

[21] Farbod, M., Akbarizadeh, G., Kosarian, A., and Rangzan, K.: "Optimized fuzzy cellular automata for synthetic aperture radar image edge detection”, J. Electron. Imag., 2018, 27 (1), 013030.

[22] Modava, M., Akbarizadeh. G., and Soroosh, M., "Integration of Spectral Histogram and Level Set for Coastline Detection in SAR Images", IEEE Transactions on Aerospace and Electronic Systems , 2019, 55(2), pp. 810-819
[23] Raeisi, A., and Akbarizadeh, G. and Mahmoudi, A.: "Combined Method of an Efficient Cuckoo Search Algorithm and Nonnegative Matrix Factorization of Different Zernike Moment Features for Discrimination Between Oil Spills and Lookalikes in SAR Images", IEEE Journal of Selected Topics in Applied Earth Observations and Remote Sensing, Nov. 2018., 11, (11), pp. 4193 4205 .

[24] Irshad, M., Sharif, M., \& Yasmin, M., and Khan, A.: "A Survey on Left Ventricle Segmentation Techniques in Cardiac Short Axis MRI", Current Medical Imaging Reviews, 2018, 14,( 2), pp. 223-237.

[25] Peng, P., Lekadir, K., Gooya, A., Shao, L., Petersen, S. E., and Frangi, A. F.: "A review of heart chamber segmentation for structural and functional analysis using cardiac magnetic resonance imaging”, Magma, 2016, 29(2), pp155-95.

[26] Goshtasby, A. and Turner, D. A.: "Segmentation of cardiac cine MR images for extraction of right and left ventricular chambers", IEEE Trans. Med. Imaging, Mar. 1995, 4, (1), pp. 5664.

[27] Nachtomy, E., Cooperstein, R., Vaturi, M., Bosak, E., Vered, Z., and Akselrod, S.: "Automatic assessment of cardiac function from short-axis MRI: procedure and clinical evaluation”, Magn. Reson. Imaging, May 1998, 16, (4), pp. 365-376.

[28] Katouzian, A., Prakash, A., and Konofagou, E.: “A New Automated Technique for Left- and Right-Ventricular Segmentation in Magnetic Resonance Imaging”, IEEE Eng. Med. Biol. Soc. Conf., Feb. 2006, 1, pp. 3074-7.

[29] Lin, X., Cowan, B. R. and Young, A. A.: "Automated detection of left ventricle in 4D MR images: experience from a large study", MICCAI Int. Conf. Med. Image Comput. and Comput.-Assist. Interv., 2006,9, (1), pp. 728-735.

[30] Geiger, D. Gupta, A. Costa, L. A. and Vlontzos, J.: "Dynamic programming for detecting, tracking, and matching deformable contours", IEEE Trans. Pattern Anal. Mach. Intell., Mar. 1995, 17, (3), pp. 294-302.

[31] Lalande, A., Legrand, L., Walker, P.M., Jaulent, M.C., Guy, F., Cottin, Y. and Brunotte F.: "Automatic detection of cardiac contours on MR images using fuzzy logic and dynamic programming", Proc. Conf. Am. Med. Inform. Assoc. Fall Symp., 1997, pp. 474-478.

[32] Liu, N., Strugnell, W., Slaughter, R., Riley, R., Crozier, S., Wilson, S., Liu, F., Appleton, B., Trakic, A. and Wei, Q.: "Right Ventricle Extraction by Low level and Model-based algorithm", IEEE Engineering in Medicine and Biology, 2005, pp. $1607-1610$.

[33] Jolly, M. P., Xue, H., Grady, L. and Guehring, J.: "Combining registration and minimum surfaces for the segmentation of the left ventricle in cardiac cine MR images.", Med. Image Comput. and Comput.-Assist. Interv., 2009, (12), (2), pp. 910-918.

[34] Cousty, J., Najman, L., Couprie, M., Clément-Guinaudeau, S., Goissen, T., and Garot, J.: "Segmentation of 4D cardiac MRI: Automated method based on spatio-temporal watershed cuts", Image Vis. Comput, 2010, 28, pp. 1229-1243.

[35] Mahmoudi, R., Akil, M., and Bedoui, M. H.: "Concurrent computation of topological watershed on shared memory parallel machines", Parallel Computing., 2017, 69, pp. 78-97.

[36] Lu, Y.-L., Connelly, K. A., Dick, A. J., Wright, G. A. and Radau, P. E. "Automatic functional analysis of left ventricle in cardiac cine MRI," Quant. Imaging Med. Surg., vol. 3, no. 4, pp. 200-209, Aug. 2013. 
[37] Waiter, G. D., McKiddie, F. I., Redpath, T. W., Semple, S. I. and Trent, R. J. "Determination of normal regional left ventricular function from cine-MR images using a semi-automated edge detection method," Magn. Reson. Imaging, Jan. 1999, vol. 17, no. 1, pp. 99-107.

[38] Lee, H.-Y., Codella, N., Cham, M., Prince, M., Weinsaft, J. and Wang, Y. "Left ventricle segmentation using graph searching on intensity and gradient and a priori knowledge (lvGIGA) for short-axis cardiac magnetic resonance imaging”, Journal Magn. Reson. Imaging, Dec. 2008, 28, (6), pp. 1393-1401.

[39] Hadhoud, M. M. A., Eladawy, M. I., Farag, A., Montevecchi, F. M. and Morbiducci, U.: "Left Ventricle Segmentation in Cardiac MRI Images", Am. J. Biomed. Eng., (2012), 2, 3, pp. 131135 .

[40] Cocosco, C. A., Netsch, T., Sénégas, J., Bystrov, D., Niessen, W. J. and Viergever, M. A: "Automatic cardiac region-ofinterest computation in cine 3D structural MRI", Int. Congr. Ser., 2004,6, (1268), pp. 1126-1131.

[41] Stalidis, G., Maglaveras, N., Efstratiadis, S. N., Dimitriadis, A. S. and Pappas, C. : "Model-based processing scheme for quantitative 4-D cardiac MRI analysis", IEEE Trans. Inf. Technol. Biomed., Mar. 2002, 6, (1), pp. 59-72.

[42] Kedenburg, G., Cocosco, C. A., Köthe, U., Niessen, W. J., Vonken, P. A., and Viergever, M. A.: "Automatic cardiac MRI myocardium segmentation using graphcut", Proc. SPIE - Int. Soc. Opt. Eng., Mar. 2006, vol. 6144.

[43] Papademetris, X., Sinusas, A. J., Dione, D. P., Constable, R. T. and Duncan, J. S.: "Estimation of 3-D left ventricular deformation from medical images using biomechanical models", IEEE Trans. Med. Imaging, Jul. 2002, 21, (7), pp. 786-800.

[44] Santarelli, M. F., Positano, V., Michelassi, C., Lombardi, M. and Landini, L.: "Automated cardiac MR image segmentation: theory and measurement evaluation", Med. Eng. Phys., Mar. 2003, 25, (2), pp. 149-159.

[45] Heiberg, E., Wigstrom, L., Carlsson, M., Bolger, A. F. and Karlsson, M.: "Time-resolved three-dimensional automated segmentation of the left ventricle", Computers in Cardiology, Lyon, France, 2005, pp. 599-602.

[46] Lynch, M., Ghita, O., and Whelan, P. F.: "Automatic segmentation of the left ventricle cavity and myocardium in MRI data", Comput. Biol. Med., Apr. 2006, 36, pp. 389-407.

[47] Sun, W., Cetin, M., Chan, R., Reddy, V., Holmvang, G., Chandar, V., and Willsky, A.: "Segmenting and Tracking the Left Ventricle by Learning the Dynamics in Cardiac Images", Information Processing in Medical Imaging, CO, USA, July 2005, pp. 553-565.

[48] Khalifa, F., Beache, G. M., Elnakib, A., Sliman, H., Gimel'farb, G., Welch, K. C., and El-Baz, A.: "A new shape-based framework for the left ventricle wall segmentation from cardiac first-pass perfusion mri”, IEEE Inter. Sym. on Biomed. Imaging, San Francisco, USA, April 2013, pp. 41-44.

[49] Ngo T. A. and Carneir, G.: "Left ventricle segmentation from cardiac MRI combining level set methods with deep belief networks", IEEE Int. Conf. Image Process., 2013, pp. 695-699.

[50] Zhang, H., Wahle, A., Johnson, R. K., Scholz, T. D. and Sonka, M.: "4-D cardiac MR image analysis: left and right ventricular morphology and function", IEEE Trans. Med. Imaging, Feb. 2010, 29, (2), pp. 350-364.

[51] Inamdar, R. S. and Ramdasi, D. S.: "Segmentation of Short Axis Cardiac MR Images using PCA with Active Appearance
Model", IOSR Journal of Computer Engineering, 2012,7, (6), pp. 25-30.

[52] Lelieveldt, B., Van Der Geest, R., Reiber, J., Bosch, J. G., Mitchell, S. and Sonka, M.: "Time-Continuous Segmentation of Cardiac Image Sequences Using Active Appearance Motion Models”, Inter. Congress Series, 2001, 1230, pp. 446-452.

[53] Zambal, S., Hladuvka, J., and Bühler, K.: "Improving Segmentation of the Left Ventricle Using a Two-Component Statistical Model", Medical Image Computing and ComputerAssisted Intervention, 2006, pp. 151-158.

[54] Lorenzo-Valdés, M., Sanchez-Ortiz, G. I., Elkington, A. G., Mohiaddin, R. H. and Rueckert, D.: "Segmentation of 4D cardiac MR images using a probabilistic atlas and the EM algorithm", Med. Image Anal., Sep. 2004, 8 (3), pp. 255-265.

[55] Lötjönen, J., Kivistö, S., Koikkalainen, J., Smutek, D. and Lauerma, K.: "Statistical shape model of atria, ventricles, and epicardium from short- and long-axis MR images", Med. Image Anal., 8, (3), Sep. 2004, pp. 371-386.

[56] Zhuang, X., Hawkes, D. J., Crum, W. R., Boubertakh, R., Uribe, S., Atkinson, D., Batchelor, P., Schaeffter, T., Razavi, R. , and Hill, D. L. G.: "Robust registration between cardiac MRI images and atlas for segmentation propagation", SPIE Medical Imaging - Image Processing, 691408, San Diego, California, March 2008.

[57] Kong, B., Zhan, Y., Shin, M., Denny, T., and Zhang, S.: "Recognizing end-diastole and end-systole frames via deep temporal regression network", In International conference on medical image computing and computer-assisted intervention, 2016, pp. 264-272.

[58] Emad, O., Yassine, I., and Fahmy, A.: "Automatic localization of the left ventricle in cardiac mri images using deep learning", In 37th Annual International Conference of the IEEE Engineering in Medicine and Biology Society (EMBC),2015, pp. 683-686.

[59] Zhang, L., Gooya, A., Dong, B., Hua, R., Petersen, S., Medrano-Gracia, P., and Frangi, A.: "Automated quality assessment of cardiac $\mathrm{mr}$ images using convolutional neural networks", In International Workshop on Simulation and Synthesis in Medical Imaging, 2016, pp. 138-145.

[60] Yang, H., Sun, J., Li, H., Wang, L., and Xu, Z.: "Deep fusion net for multi-atlas segmentation: Application to cardiac mr images", In International Conference on Medical Image Computing and Computer-Assisted Intervention, 2016, pp. 521-528.

[61] Ngo, T., Lu, Z., and Carneiro, G.: "Combining deep learning and level set for the automated segmentation of the left ventricle of the heart from cardiac cine magnetic resonance", Journal of Medical image analysis, 2017, (35), pp. 159-171.

[62] Rupprecht, C., Huaroc, E., Baust, M., and Navab. N.: "Deep active contours", Computing Research Repository, 2016, (1607)05074.

[63] Poudel, R., Lamata, P., and Montana, G.: "Recurrent fully convolutional neural networks for multi-slice mri cardiac segmentation" In Reconstruction, segmentation, and analysis of medical images, 2017, pp. 83-94.

[64] Oktay, O., Bai, W., Lee, M., Guerrero, R., Kamnitsas, K., Caballero, J., Marvao, A., Cook, S., O’Regan, D. and Rueckert. D.: "Multi-input cardiac image super-resolution using convolutional neural networks" In International conference on medical image computing and computerassisted intervention, 2018, pp. 246-254. 
[65] Berbari, R. : "Segmentation of contraction images and late enhancement in cardiac MRI : Study of contractile function and myocardial viability", PhD thesis, Télécom ParisTech, 2009.

[66] Constantinides, C. : “ Automated segmentation of the left ventricle in cardiac MRI: A comparative evaluation of supervised and unsupervised approaches for myocardial viability studies", PhD thesis, ENST, 2012.

[67] Haggerty, C. M., Kramer, S. P., Skrinjar, O., Binkley, C. M., Powell, D. K., Mattingly, A.C., Epstein FH, Fornwalt BK.: "Quantification of left ventricular volumes, mass, and ejection fraction using cine displacement encoding with stimulated echoes (DENSE) MRI", Journal of magnetic resonance imaging, 2013, 40(2), pp. 398-406.

[68] Borzage, M., Heidari, K., Chavez, T., Seri, I., Wood, J.C. and Blüml, S.: "Measuring Stroke Volume: Impedance Cardiography vs Phase-Contrast Magnetic Resonance Imaging", American Journal of Critical Care, Sep. 2017, 26(5): pp. 408-415

[69] Foley T.A., Mankad S.V., Anavekar N.S., Bonnichsen C.R., Morris M.F., Miller T.D., Araoz P.A. : "Measuring left ventricular ejection fraction-techniques and potential pitfalls", European Cardiology, 2012, 8 (2), pp. 108-114.

[70] García, X., Mateu, L., Maynar, J., Mercadal, J., Ochagavía, A. and Ferrandiz, A.: "Estimating cardiac output : Utility in the clinical practice, Available invasive and non-invasive monitoring", Med Intensiva. Dec. 2011, 35(9), pp.552-61

[71] Foppa, M., Duncan, B. B., \& Rohde, L. E.: "Echocardiography-based left ventricular mass estimation. How should we define hypertrophy? ", Cardiovascular ultrasound, 2005, (3)17.

[72] Chengode, S.: "Left ventricular global systolic function assessment by echocardiography", Annals of cardiac anaesthesia", 2016, 19 (5), pp 26-34

[73] Bacharach, S.L., Green, M.V., Borer, J.S., Hyde, J.E., Farkas, S.P. and Johnston, G.S.: " Left-ventricular peak ejection rate, filling rate, and ejection fraction--frame rate requirements at rest and exercise: concise communication", Journal of Nuclear Medicine, Mar. 1979, 20(3):pp. 189-93.

[74] Feng, W., Nagaraj, H., Gupta, H., Lloyd, S. G., Aban, I., Perry, G. J., Calhoun, D., A., Dell'Italia, J. L. and Denney, T. S.: "A dual propagation contours technique for semi-automated assessment of systolic and diastolic cardiac function by CMR", Journal of Cardiovascular Magnetic Resonance, 2009, 11(1).

[75] Petersen, S. E., Voigtländer, T., Kreitner, K. F., Horstick, G., Ziegler, S., Wittlinger, T., Abegunewardene, N., Schmitt, M., Schreiber, W. G., Kalden, P., Mohrs, O. K., Lippold, R., Thelen and M., Meyer, J.: " Late improvement of regional wall motion after the subacute phase of myocardial infarction treated by acute PTCA in a 6-month follow-up", Journal of Cardiovascular Magnetic Resonance, Jul. 2003,5(3), pp. 487-95.

[76] Pearlman, P. C., Tagare, H. D., Lin, B. A., Sinusas, A. J. and Duncan, J. S.: "Segmentation of 3D RF Echocardiography Using a Multiframe Spatio-temporal Predictor", Information Processing in Medical Imaging. Lecture Notes in Computer Science, vol. 6801. Springer, Berlin, Heidelberg, 2011.

[77] Hartley, Herman O.: "The Maximum F-ratio As a Short-cut Test for Heterogeneity of Variance", Biometrika, 1950, 37(3/4), pp. 308-312.

[78] David, Hartley A.: "Upper 5 and 1\% Points of the Maximum F-ratio.” Biometrika, 1952, 39(3/4), pp.422-424.

[79] Benameur, N., Caiani, E.G., Arous, Y., Abdallah, NB., and Kraiem, T.: "Interpretation of Cardiac Wall Motion from Cine-
MRI Combined with parametric Imaging based on the Hilbert transform", Magn Reson Mater Phys 2017,(30),pp.347-57.

[80] Ammari, A., Mahmoudi, R., Saouli, R., Hmida, B. and Bedoui, M. H.: "A collection study of the approaches investigated for Right Ventricular segmentation in CMRI", Applications Medicales de l'Informatique Nouvelles Approches - AMINA Workshop, Nov. 2018, 9, pp. 122-132. 